\section{DOHaD et information épigénétique}

\section{Enjeux sociétaux}

\author{
Emmanuelle Rial-Sebbag ${ }^{1}$, Caroline Guibet Lafaye ${ }^{2}$, \\ Umberto Simeoni ${ }^{3}$, Claudine Junien ${ }^{4}$
}

developmental origins of health and disease) modifie notre compréhension de ce qui fait qu'un individu développe, au cours de sa vie, les principales maladies chroniques qui affectent les pays riches ou émergents, ou reste en bonne santé. Il implique une évolution du paradigme sur lequel peuvent s'appuyer les politiques de prévention à l'échelle de la planète, dans le champ majeur de l'épidémiologie et des mécanismes des maladies non transmissibles selon la classification de l'Organisation mondiale de la santé. Ce concept ouvre une vision nouvelle également dans les sciences psychologiques, sociales, économiques, éthiques et juridiques. Ce ne sont pas uniquement les aspects épigénétiques qui sont appréhendés par les sciences humaines et sociales mais également les conséquences sociales produites par le développement des connaissances liées à la DOHaD et notamment les politiques publiques qui pourraient être mises en œuvre. Les informations révélées par l'épigénétique, couplées aux informations sur le style de vie et à celles recueillies durant le développement, sont d'un genre nouveau, proche mais différent des informations génomiques, faisant dès lors apparaître des enjeux de nature différente. Elles méritent, de ce fait, d'être accompagnées par une réflexion pluridisciplinaire dès l'origine de leur production afin d'anticiper les questionnements qui pourraient être soulevés dans le futur. <

\section{Le concept DOHaD : un impact en santé publique et santé individuelle}

Le concept de l'origine développementale de la santé et des maladies ( $\mathrm{DOHaD}$ ) transforme notre compréhension des origines d'un état de bonne santé et des facteurs

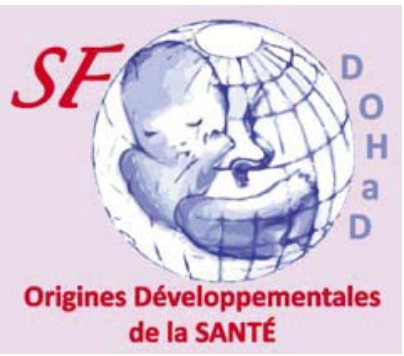

de risque de nombreuses maladies chroniques acquises, en particulier les maladies cardio-vasculaires, le diabète de type 2, l'obésité. L'hypothèse selon laquelle des maladies chroniques de l'âge adulte ont, au moins partiellement, une origine précoce, au cours du développement, implique une évolution des paradigmes sur lesquels s'appuient les politiques de prévention à l'échelle globale. Elle ouvre également une vision nouvelle dans le champ des sciences psychologiques, sociales, économiques, éthiques et juridiques.

La fréquence croissante et la gravité des maladies non transmissibles (MNT) acquises avec l'âge constituent un défi majeur sur le plan humain et économique pour les pays riches et, surtout, pour les pays à faibles ressources. L'orientation actuelle des actions de santé publique, centrées sur la maladie, en particulier l'obésité, appelle donc un changement de paradigme qui intègre une nouvelle dimension de prévention appuyée sur le concept «DOHaD ». Cette nouvelle approche met l'accent sur l'importance des facteurs environnementaux (l'environnement est entendu ici au sens large : environnement naturel, utérin ou encore social) affectant les périodes les plus précoces du développement et influençant plus tard, à l'âge adulte, des réponses adaptées ou inadéquates à un environnement délétère $[1](\rightarrow)$.

De nombreuses données scientifiques suggèrent qu'il existe un cercle vicieux impliquant, dans

$\rightarrow$ Voir l’éditorial de M.N. Bruné Drisse, page 9 de ce numéro le cycle de la reproduction, les conséquences des expositions, des modes de vie et des comportements au cours de fenêtres précoces de vulnérabilité pendant la période péri-conceptionnelle, la grossesse et la première enfance, contribuant à la diffusion de certaines maladies 
chroniques de génération en génération. Le mode de vie du père et de la mère [2], leur état nutritionnel et métabolique (en particulier l'existence d'un surpoids, d'une obésité ou d'un diabète de type 2), leur contexte psychologique et statut socioéconomique (SSE) à la conception [3], pendant la grossesse, et l'environnement exercent une influence à long terme sur les principales régulations fonctionnelles de l'enfant en développement. Des mécanismes épigénétiques sont impliqués dans certains effets intergénérationnels ou transgénérationnels. Cependant, le déchiffrage des mécanismes épigénétiques complexes n'en est qu'à ses débuts. Certaines mesures comme l'allaitement maternel, des conseils portant sur la diététique et l'activité physique [4] $(\rightarrow)$, la réduction de l'exposition à des substances toxiques de l'environnement durant la phase précoce de sensibilité [5] peuvent contribuer à réduire l'incidence de ces affections.

Les mécanismes de la DOHaD sous-tendent non

\section{$(\rightarrow)$ Voir les 2 Synthèses de C. Junien et al., pages 27 et 35 de ce numéro} seulement le champ de la pathologie, mais aussi celui des variations biologiques à l'intérieur de ce qui est considéré comme les limites de la normalité. Celles-ci pourraient, «à génome constant», être expliquées par des marques épigénétiques perturbées par les variations de l'environnement dans lequel se déroule le développement précoce de l'individu. Les marques épigénétiques (méthylation de l'ADN, modifications des histones, ARN non codants), sont mises en place tout au long de la vie, mais semblent particulièrement sensibles au moment où le développement, la différenciation cellulaire, l'organogenèse, la maturation fonctionnelle interviennent, ces phénomènes impliquant normalement des mécanismes permettant l'activation ou l'inhibition des gènes. Ces stades sont particulièrement sensibles aux impacts de l'environnement.

L'impact potentiel de la DOHaD, dans le domaine de la santé globale entendue au sens large, comme un état de bien-être physique, social et mental ${ }^{1}$ mais aussi sur le plan économique et social, est donc conséquent. En effet les maladies chroniques (hypertension artérielle, obésité, diabète, cancers, allergies, etc.) explosent à travers le monde : $60 \%$ des décès, 35 millions de morts par an dont $80 \%$ dans les pays à faible ou moyen revenu.

Cet impact affecte les pays disposant de ressources élevées, touchés par un mode de vie à forte consommation et historiquement par les maladies dites de civilisation. Ces maladies comprennent les maladies cardiovasculaires, du métabolisme, auto-immunes et de l'immunité, et les maladies dégénératives. Ce profil de maladies est très différent dans les pays en voie de développement, dans lesquels cinq maladies infectieuses (pneumonies, diarrhées, rougeole, paludisme et Sida) et la malnutrition sont à l'origine de plus de la moitié des décès des enfants de moins de cinq ans. Cependant, et de façon croissante, l'impact potentiel de la DOHaD concerne également les pays à niveau de ressources moyen ou faible, dans lesquels les projections de la part

${ }^{1}$ La santé est un état de complet bien-être physique, mental et social, et ne consiste pas seulement en une absence de maladie ou d'infirmité. Préambule à la Constitution de l'Organisation mondiale de la santé, tel qu'adopté par la Conférence internationale sur la santé, New York, 19-22 juin 1946 ; signé le 22 juillet 1946 par les représentants de 61 États (Actes officiels de l'Organisation mondiale de la santé, $n^{\circ} 2$, p. 100) et entré en vigueur le 7 avril 1948. liée aux maladies chroniques dans la mortalité totale sont préoccupantes [6].

Le retour sur investissement des politiques de prévention des maladies chroniques (MC), anciennement dénommées non transmissibles (MNT), de l'adulte est connu pour augmenter de façon quasi-exceptionnelle lorsque les actions préventives sont orientées vers les phases les plus précoces de la vie, dans l'enfance et davantage encore durant la période périnatale et la grossesse, par opposition aux actions ciblant les sujets adultes. De nombreuses organisations non gouvernementales mais aussi les pouvoirs publics se sont tournés vers le concept dit «des 1000 jours » pour représenter la période s'étendant de la conception à la fin de la deuxième année de vie de l'enfant, y compris dans le champ de la lutte contre la précarité et dans le développement durable 2 .

Ainsi, à la conception binaire classique des causes des MNT, combinant le rôle de l'environnement et du style de vie à l'âge adulte et un terrain génétique prédisposant, vient se substituer une représentation triple incluant un troisième pilier, l'épigénome, constitué durant le développement sous l'effet de l'environnement précoce. Des études de cohorte ont montré que, pour ce qui est du risque d'hypertension artérielle à l'âge adulte, le rôle de l'environnement durant les phases précoces du développement (révélé par le poids à la naissance) est comparable, en termes d'ampleur d'effet, à celui de l'environnement à l'âge adulte. D'autre part, le rôle du génotype ne semble pas dépasser $25 \%$ [7] dans les estimations les plus favorables, les $75 \%$ restants étant associés à des interactions entre génotype et environnement, donc à des mécanismes relevant de l'épigénétique et imputable au phénomène de DOHaD.

Dans ce contexte, ce n'est pas tant le mécanisme épigénétique qui est appréhendé par les sciences humaines et sociales que les conséquences sociales qui pourraient être induites par les connaissances liées au concept DOHaD. En particulier, de nouveaux risques pourraient apparaître, notamment de discrimination envers les femmes qui sont le plus souvent les sujets des études produites dans le domaine [8]. Les informations produites par l'épigénétique, couplées à celles sur le style de vie, en particulier durant le développement, sont d'un genre nouveau pouvant s'entendre tant au sens biologique (les informations épigénétiques s'inscrivent dans la continuité des informations génétiques en ce que l'information épigénétique correspond à des marques imprimées sur le génome influençant son expression), qu'au sens individuel (les modifications

\footnotetext{
${ }^{2}$ www.thousanddays.org
} 
épigénétiques engendrées par l'environnement, également dénommées épimutations, sont des informations personnelles). Cependant, ces marques flexibles et dynamiques sont considérées comme pouvant être réversibles, suggérant de possibles dispositifs de prévention afin d'éviter leur apparition ou encore de réduire leur transmission. Pourtant, la connaissance des mécanismes épigénétiques n'est pas encore scientifiquement stabilisée (leur transmission et leurs conséquences sur la santé sont encore à l'étude) empruntant dès lors à plusieurs registres de valeurs, de normes et de responsabilités. Devant cette instabilité scientifique, il est nécessaire d'anticiper les enjeux sociétaux qui pourraient survenir au fur et à mesure du développement des connaissances grâce à la mise en œuvre d'une approche $\varepsilon L S I$ « by design $»^{3}$ (ethical, legal and social issues). Dans un contexte d'incertitude scientifique, la démarche «by design » permet d'accompagner le développement des connaissances et de produire différents scénarios dans la prise de décision. Cette approche est également considérée comme une inflexion du paradigme de la réflexion bioéthique qui, plutôt que de s'inscrire dans une approche a priori, intervenait plus classiquement a posteriori. Ainsi le recours à l'information épigénétique comme modèle explicatif engage à s'interroger sur son régime juridique ainsi que sur certains principes éthiques qui pourraient être remis en question à l'échelle individuelle. Cette information fait également émerger de nouvelles collaborations, notamment entre économie et nutrition, afin d'élaborer de meilleures stratégies de santé publique, basées en partie sur les informations recueillies dans le cadre du concept DOHaD.

\section{L’information épigénétique : quelle régulation?}

Appréhender l'information épigénétique du point de vue de la loi, nécessite d'en définir la nature afin d'en déterminer le régime juridique. Il pourrait aller de soi d'associer information épigénétique et information génétique, afin de les soumettre à un régime commun tant les deux sciences dont elles sont issues ${ }^{4}$ [9] sont liées et complémentaires $(\rightarrow)$.

Cependant les caractéristiques des marques et des

$(\rightarrow)$ Voir la Synthèse de C. Junien et al., page 35 de ce numéro mécanismes épigénétiques font que le questionnement juridique qu'elles soulèvent diffère de celui concernant l'information génétique. Pour cette dernière, il faut en effet distinguer les affections génétiques monogéniques qui confèrent la maladie, des affections polygéniques pour lesquelles la plupart des variants génétiques ne confèrent individuellement qu'une faible prédisposition à une maladie, souvent chronique d'ailleurs. Si, par nature, les marques épigénétiques sont dynamiques, en revanche, les conséquences de leurs changements, sur la morphogenèse ou sur l'état de la cellule, sont parfois irréversibles. Les marques épigénétiques

\footnotetext{
${ }^{3}$ Cette approche by design a été initialement développée dans le contexte de la protection de la vie privée au regard du développement des nouvelles technologies de l'information et consiste à intégrer en amont, dès la conception des outils, des éléments de protection des droits fondamentaux des individus. Résolution sur la Protection intégrée de la vie privée, $32^{\mathrm{e}}$ Conférence internationale des commissaires à la protection des données et de la vie privée, Jérusalem, 2010, https://www.ipc.on.ca/site_documents/ pbd-resolution-f.pdf

${ }^{4}$ L'épigénétique correspond à « l'adaptation structurelle de régions chromosomiques permettant d'enregistrer, de signaler ou de perpétuer des états d'activité modifiés » (A. Bird [9]).
}

qui accompagnent un tel conditionnement délétère au cours du développement sont associées à une susceptibilité qui rend l'individu qui en est porteur plus sensible à un environnement inadéquat mais ne confèrent pas un risque systématique de maladie. S'engager sur la voie de l'analogie reviendrait alors à soumettre l'information épigénétique à un régime restrictif, particulièrement rigoureux tel qu'il est prévu pour l'information génétique monogénique. Cette dernière ne dispose pas de réelle définition légale en France ${ }^{5}$ mais bénéficie d'ores et déjà de principes d'encadrement spécifiques et ce pour deux raisons principales. Premièrement, comme elle concerne des caractéristiques transmissibles, certains membres de la famille ont un intérêt personnel à connaître l'existence de la mutation responsable de la maladie pour leur permettre d'être dépistés et éventuellement traités ou pour prendre des décisions concernant la procréation. Deuxièmement, le génome, entendu comme élément constitutif de la personne, est par définition identifiant. De ce fait, la possibilité d'avoir recours à l'utilisation des informations génétiques dans un but de discrimination n'est pas exclue, notamment dans le cadre de l'emploi [10] ou des assurances [11]. Ces caractères particuliers sont ceux qui ont justifié l'adoption de dispositions juridiques spécifiques en France où l'information génétique est considérée comme une information de santé individuelle particulière ${ }^{6}$. Son traitement par la norme juridique relève soit de la règlementation encadrant le soin (diagnostic et/ou thérapeutique), soit de celle relevant de la recherche, mais, dans les deux cas, son régime est dérogatoire du droit commun. Notamment les dispositions de protection des personnes dont sont issues les informations génétiques sont plus rigoureuses et commandent un encadrement plus strict en termes d'information et de recueil du consentement ${ }^{7}$, ou impliquent des règles strictes de confidentialité du fait de ses potentialités identifiantes. Mais ces différentes mesures sont-elles applicables mutatis mutandis à l'information épigénétique? Si l'information épigénétique peut être entendue comme «toutes informations issues de la mise en évidence d'interactions entre biologie et

\footnotetext{
${ }^{5}$ Seules les données issues de prélèvements biologiques identifiants sont visées par la Loi informatique et libertés à son article 56 (Loi 78-17 du 6 janvier 1978 modifiée).

${ }^{6}$ La notion d'information de santé ne dispose pas à ce jour de réelle définition juridique. On peut toutefois se référer à la définition donnée par la proposition de règlement en passe d'être adoptée par l'Union Européenne, Art. 4-12 de la proposition «données concernant la santé » : toute information relative à la santé physique ou mentale d'une personne, ou à la prestation de services de santé à cette personne, Proposition de Règlement du Parlement Européen et du Conseil relatif à la protection des personnes physiques à l'égard du traitement des données à caractère personnel et à la libre circulation de ces données (règlement général sur la protection des données), COM (2012) 2011 final.

${ }^{7}$ Article 16-10 du code civil, Tout examen des caractéristiques génétiques requiert
} le recueil d'un consentement par écrit. 
environnement délivrant des renseignements sur l'individu, son histoire personnelle et sa santé » [12] alors l'information épigénétique partage certains critères avec l'information génétique [13] : un certain degré de transmissibilité et un potentiel d'identification des individus. Cette potentialité a d'ailleurs été récemment discutée dans le contexte de la protection de la vie privée et de la confidentialité à propos de la possible ré-identification de patients à partir de leurs données épigénétiques. Si les risques de ré-identification individuels sont réels (information sur les personnes mais également informations sur d'éventuelles conduites à risques) [14], leur réalisation semble toutefois plus hypothétique [15] et le risque devra être, quoiqu'il en soit, réévalué à la lumière des nouvelles techniques de séquençage à haut débit. Ces dernières sont d'ores et déjà à l'œuvre dans le domaine de l'épigénétique (voir les données obtenues par l'IHEC [international human epigenome consortium] qui a mis en ligne, en mars 2015, 5500 jeux de données épigénétiques, http:// ihec-epigenomes.org/) et conduisent à des mesures d'accès limité, pour les chercheurs, afin de garantir au maximum la sécurité de ces données et de réduire le risque d'atteinte à la vie privée des patients. «L'information épigénétique nous renseigne (certes) sur une personne (mais) dans son contexte » [12]. C'est pourquoi la dimension collective et de santé publique reste prédominante pour les enjeux de régulation. Cependant l'encadrement juridique devra davantage porter sur l'analyse des facteurs environnementaux influençant l'information épigénétique que sur la protection individuelle plus classiquement étudiée pour l'information génétique [16]. Deux scénarios ont été envisagés ${ }^{8}$ : soit une inclusion de l'information épigénétique dans le régime juridique de l'information génétique (la difficulté sera ici de justifier un régime restrictif du droit commun qui risquerait de s'analyser comme une démarche « exceptionnaliste ») ; soit l'adoption d'un régime juridique propre. Une troisième voie, celle d'un rattachement de l'information épigénétique au régime plus souple des informations de santé, adossé à un renforcement des responsabilités collectives (en matière de droit du travail ou de droit de l'environnement ou encore de mise en œuvre de programmes de prévention), pourrait de plus être explorée. Ce sont alors des enjeux de gouvernance et de santé publique qui prendront ici le pas sur le colloque singulier (médecin-patient) bien connu dans le champ de la génétique médicale. Les acteurs publics se trouveront au cœur de la décision et des responsabilités collectives concernant le bien-fondé de l'usage de cette information épigénétique en santé, bien que nous ne devions pas ignorer dans un futur proche, de probables mises en jeu de responsabilités individuelles ${ }^{9}$ (plus précisément intra-familiales). Au-delà de la responsabilité juridique pouvant être potentiellement

\footnotetext{
${ }^{8}$ Office Parlementaire des choix scientifiques et techniques, Etude de faisabilité de la saisine sur «Les enjeux et les perspectives de l'épigénétique», 26 mai 2015, http://www.assemblee-nationale.fr/opecst/ faisabilite_epigenetique.pdf

${ }^{9}$ L'état du Tenessee (États-Unis) vient d'adopter une législation permettant de poursuivre une femme enceinte qui aurait causé des dommages durant sa grossesse au fœtus du fait d'un comportement addictif : http://www.salon.com/2014/04/30/tennessee_just_became_the_first_state_that_will_jail_ women_for_their_pregnancy_outcomes/ ; a contrario, en France, en mars 2009, le tribunal correctionnel de Chartres a relaxé une mère poursuivie pour absence de suivi de sa toxicomanie durant sa grossesse et blessures involontaires sur l'enfant auquel elle a donné naissance qui est né avec un lourd handicap. Dans cette dernière affaire le tribunal a conclu à la relaxe du fait qu'on ne pouvait étendre les comportements à risques à des fautes pénales. Cependant cette décision, favorable à l'absence de qualification pénale, montre d'une part que les poursuites peuvent être engagées et d'autre part, comme décision rendue par les juges du fond, elle pourrait être susceptible d'être jugée différemment par le juge de la cassation.
}

engagée, une responsabilité plus morale de la gestion de cette information pourra également être prise en compte.

\section{L’information épigénétique : quels enjeux éthiques?}

La mise en évidence et la communication d'informations liées à des processus épigénétiques soulèvent des questions éthiques, tenant notamment à l'usage de ces informations dans des politiques de santé, à l'usage de données d'ordre épigénétique par les compagnies d'assurance ou encore à l'éthique de la communication d'informations $(\rightarrow)$. Ne sera ici abordée que la question de l'exposition individuelle, non souhaitée, à une information impli-

$(\rightarrow)$ Voir $m / s$, hors série $n^{\circ} 2$, juin-juillet 2015

quant des risques en matière de santé, pour soi-même et pour sa descendance. Dans le cas de la génétique comme de l'épigénétique, la recherche d’informations sur des données privées concerne aussi bien l'individu que ses ascendants. Elle est signifiante non pas seulement pour ego (soi-même) mais également pour ses descendants.

Dans le domaine juridique, le droit à une information claire et proportionnée ainsi qu'un droit de ne pas savoir sont affirmés [17, 18]. Ce droit de ne pas savoir, indissociable du droit d'une personne à connaître toute information recueillie sur sa santé, est énoncé dans l'article 10, § 2 de la Convention sur les droits de l'homme et la biomédecine : «Toute personne a le droit de connaître toute information recueillie sur sa santé. Cependant, la volonté d'une personne de ne pas être informée doit être respectée. » II est vrai que l'alinéa 3 ajoute qu' « À titre exceptionnel, la loi peut prévoir, dans l'intérêt du patient, des restrictions à l'exercice des droits mentionnés au paragraphe $2 »$. Le Code de la santé publique (CSP), en France, fait écho à cette disposition : «lorsqu'une personne demande à être tenue dans l'ignorance d'un diagnostic ou d'un pronostic, sa volonté doit être respectée, sauf si des tiers sont exposés à un risque de contamination. [...] » (Code de déontologie médicale, Titre II, art. 35 ; article R.412732 du Code de la santé pubique). À côté du «droit de ne pas savoir » qui s'applique plus à la génétique monofactorielle, certains chercheurs défendent, du fait du caractère difficilement prédictible du phénotype, mais aussi de la possibilité de prévenir l'apparition de la maladie pour soi et sa descendance par un comportement adapté, un «devoir de savoir» où génétique multifactorielle et épigénétique s’entremêlent $(\rightarrow)$.

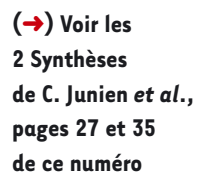


Si les individus peuvent se voir reconnaître un « droit de ne pas savoir » relatif à leur patrimoine génétique ou à des données d'ordre épigénétique, néanmoins on peut considérer - comme la loi le suggère aussi - que ce droit doit être limité. En particulier la décision de ne pas savoir peut être critiquée comme étant moralement fautive, lorsque cette volonté d'ignorance peut causer un préjudice sérieux ou un tort à une autre personne [19].

Peut-on sciemment s'opposer à la connaissance de facteurs de risque, concernant des pathologies auxquelles on peut être prédisposé, ou à la connaissance de facteurs de risque concernant sa descendance, et éventuellement les soustraire à la connaissance de sa descendance ${ }^{10}$ [20] ? La cartographie épigénétique devrait pouvoir fournir des informations sur la probabilité, pour l'individu, ainsi que pour ses descendants, d'être plus susceptible de développer certaines pathologies. La probabilité de développer une pathologie peut se décrire selon une triple modalité : facteur de risque, indicateur de risque, marqueur de risque [21]. La connaissance des probabilités et du sens qu'elles ont pour la santé individuelle et les risques qui pèsent sur celle-ci suppose une éducation et des aptitudes cognitives permettant notamment de distinguer ce qui est certain de ce qui est simplement probable. L'appréhension cognitive du risque et de la précaution est soumise à des biais caractérisés consistant à se concentrer sur certains risques, simplement parce qu'ils sont cognitivement « disponibles », alors que d'autres ne le sont pas. De plus, le «droit de ne pas savoir » a été fréquemment invoqué à propos de l'accès à l'information génétique. Il est parfois rapproché du «droit à un avenir ouvert », encore désigné sous l'expression de «droit à l'ignorance génétique » [22].

Parce que la transmission éventuelle du risque de développer une maladie chronique, que ce soit par des mécanismes épigénétiques ou par d'autres mécanismes, $(\rightarrow)$

concerne la santé des générations futures, elle

semble exiger de reconsidérer certains principes

fondamentaux de l'éthique médicale, en l'occur-

$(\rightarrow)$ Voir la Synthèse de C. Junien et al., page 35 de ce numéro rence la revendication d'un «droit de ne pas savoir » et le principe d'autonomie du patient [23]. La prise en compte d'informations relevant de l'épigénétique, dans cette perspective de santé, pèse de façon contraignante sur l'exercice de la liberté individuelle. Ainsi, rester dans l'ignorance des prédispositions génétiques à certaines maladies ne peut seulement relever de la liberté individuelle, dans la mesure où existerait une responsabilité d'ego envers sa descendance. Cette responsabilisation suppose toutefois la connaissance, par chacun, des facteurs de risque associés à certains comportements ou à certaines expositions et la conscience des coûts que l'on fait peser sur les générations présentes au nom des générations futures ${ }^{11}$.

Existent, en effet, une certaine réversibilité des prédispositions épigénétiques mais également des formes de résilience. L' « adversité » n'interdit pas la résilience susceptible de s'accompagner également de processus biologiques [24]. Les expériences en laboratoire,

${ }^{10}$ Sur la tendance à ne pas considérer qu'en épigénétique il n'est question que de facteurs de risque plutôt que d'une prédiction certaine [19].

${ }^{11}$ La notion de « coût » doit ici être comprise dans le sens d'« externalités négatives », c'est-à-dire de conséquences externes, en l'occurrence indésirables ou non souhaitées. notamment, montrent que les mêmes facteurs peuvent, dans certains contextes, protéger et, dans d'autres contextes ou dans d'autres cas, être à risque $(\rightarrow)$.

Les effets de l'environnement

précoce s'exercent aussi bien en $(\rightarrow)$ Voir la Synthèse de C. Junien et al., page 35 de ce numéro négatif qu'en positif : des soins maternels de meilleure qualité ou bien des stress modérés dans les premiers jours favorisent chez l'animal adulte la sociabilité et la résilience au stress [25]. Du fait de ces phénomènes de réversibilité et de résilience, se pose la question du rapport coûts/bénéfices de l'exposition à des informations qui, en tant que telles, sont préjudiciables pour l'individu qui en est informé voire responsabilisantes à tort. Le choix d'acquérir de l'information aussi bien que celui de s'en tenir à distance impliquent des arbitrages et comportent des coûts. Des responsabilités individuelles sont associées au «droit de savoir » aussi bien qu'au «droit de ne pas savoir ». Toutefois il est essentiel de tenir compte de ces arbitrages entre coûts et bénéfices, pour l'ensemble des parties concernées, afin de protéger les intérêts de chacune. Ces coûts concernent notamment le respect de la vie privée [26], l'exposition à des informations non souhaitées sur sa généalogie, la possibilité de contrôler la diffusion et l'usage d'informations sur soi-même, enfin les usages susceptibles d'être faits, par les assurances et les employeurs, de ces informations.

\section{Conclusion}

Pour conclure, comme le souligne J. Heckman [27], les économistes de la santé doivent désormais tenir compte non seulement des coûts et des avantages d'un traitement, mais aussi de la prévention des maladies chez les parents et tout au long du cycle de la vie. Ces coûts et avantages, de quelque nature qu'ils soient, ne se mesurent pas simplement en termes économiques car la désutilité trouve également un sens en matière de liberté individuelle, de possibilité de choix et doit s'apprécier aussi dans une perspective éthique. Les préoccupations des politiques visant à atténuer les inégalités liées au statut socioéconomique (SSE) sont de plus en plus axées sur les investissements dans la petite enfance. La majorité des interventions réussies sur la petite enfance démarrent dans les années préscolaires [2, 3]. La justification économique de l'investissement précoce devrait constituer des preuves pour la politique et les décideurs [2] dont on attend qu'ils tiennent compte de l'ensemble des coûts et des bénéfices sociaux qu'induiraient des politiques de santé, fondées sur les résultats scientifiques les plus récents 
incluant l'épigénétique. Au lieu de se contenter de décrire des effets, les scientifiques doivent donc être encouragés à produire les types de preuve en épigénétique ou pour d'autres facteurs, qui permettront d'élaborer des recommandations pour agir sur les déterminants sociaux de la santé [28]. $\diamond$

\section{SUMMARY}

DOhaD and epigenetic information: societal challenges

The concept of the developmental origins of health and disease (DOHaD) alters our understanding of what constitutes "health" or "disease" intended as chronic, non-communicable diseases, which develop over the life course in high income and emerging countries. It implies a change in paradigm forming a basis for prevention policies across the globe. It also impacts psychological, social, economic, ethical and legal sciences. In line with the unanticipated underpinning epigenetic mechanisms are also the social issues (including public policies) that could be produced by the knowledge related to DOHaD that opens a wide field of inquiry. The information unveiled by epigenetics coupled with information on lifestyle including during the development phase, is of unforeseen nature, raising issues of different nature. Therefore it requires specific attention and research, and a specific support by a pluridisciplinary reflection since the very beginning of its production, to anticipate the questions that might be raised in the future. $\diamond$

\section{LIENS D'INTÉRÊT}

Les auteurs déclarent n'avoir aucun lien d'intérêt concernant les données publiées dans cet article.

\section{REMERCIEMENTS}

Cet article a été rédigé dans le cadre du contrat IBISS (Incorporation Biologique et Inégalités Sociales de Santé, ANR-12-DSSA-0004-02, coordonnateur Cyrille Delpierre).

\section{RÉFÉRENCES}

1. Bruné Drisse MN. Environnements précoces, origines précoces de la santé et des maladies. Med Sci (Paris) $2016 ; 32: 9-10$

2. Rando 0J. Daddy issues: paternal effects on phenotype. Cell $2012 ; 151: 702-8$.

3. Borghol N, Suderman M, McArdle W, et al. Associations with early-life socio-economic position in adult DNA methylation. Int J Epidemiol $2011 ; 41: 62-74$.

4. Eckenrode J, Campa M, Luckey DW, et al. Long-term effects of prenatal and infancy nurse home visitation on the life course of youths: 19-year follow-up of a randomized trial. Arch Pediatr Adolesc Med $2010 ; 164: 9-15$.

5. Bellanger M, Pichery $C$, Aerts $D$, et al. Economic benefits of methylmercury exposure control in Europe: monetary value of neurotoxicity prevention. Environ Health $2013 ; 12: 3$.
6. Lopez AD, Mathers CD, Ezzati M, et al. Global and regional burden of disease and risk factors, 2001: systematic analysis of population health data. Lancet $2006 ; 27: 1747-57$.

7. Teh AL, Pan H, Chen L, et al. The effect of genotype and in utero environment on interindividual variation in neonate DNA methylomes. Genome Res 2014 ; $24: 1064-74$.

8. Warin M, Moore V, Zivkovic T, et al. Telescoping the origins of obesity to women's bodies: how gender inequalities are being squeezed out of Barker's hypothesis. Ann Hum Biol 2011 ; 38 : 453-60.

9. Bird A. Perceptions of epigenetics. Nature $2007 ; 447$ : 396-8.

10. Gaumont-Prat H. Tests génétiques et emploi. Propositions à l'occasion de la révision des lois de bioéthique. Médecine et Droit $2000 ; 42: 26$.

11. Gaumont-Prat H. Étude génétique des caractéristiques d'une personne et assurances. Paris : Dalloz, $2001 ; 18$ : 1429-30.

12. Anastasova $V$, Soulier $A$, Rial-Sebbag $\varepsilon$. L'information épigénétique : un nouvel objet du droit? Revue Générale de Droit Médical 2015 ; 54 : 79-91.

13. Dupras $C$, Ravitsky V, Willams-Jones $B$. Epigenetics and the environment in bioethics. Bioethics $2014 ; 28: 327-34$.

14. Philibert RA, Terry N, Erwin C, Philibert WJ, Beach SRH, Brody GH. Methylation array data can simultaneously identify individuals and convey protected health information: an unrecognized ethical concern. Clin Epigenetics 2014 ; $6: 28$.

15. Joly Y, OM Dyke S, Cheung WA, et al. Risk of re-identification of epigenetic methylation data: a more nuanced response is needed. Clin Epigenetics $2015 ; 7: 45$.

16. Rothstein MA, Cai Y, Marchant GE. The ghost in our genes: legal and ethical implications of epigenetics. Health Matrix Clevel 2009 ; 19 : 1-62.

17. Ducournau P. Droits des usagers, droits et usagers. Informations Sociales $2010 ; 158: 90-8$.

18. Moutel G, Hervé C, Grand $\varepsilon$. Les éléments du corps humain, la personne et la médecine, Paris : L’Harmattan, 2005.

19. Card R. Genetic information, health insurance, and Rawlsian justice. In : Card R, ed. Critically thinking about medical ethics. London (UK), Pearson, 2004 : 288-94.

20. Guibet Lafaye C. L'épigénétique : pour de nouvelles politiques de santé ? Humanistyka i Przyrodoznawstwo 2014 ; 20 : 306-31.

21. Bail JN, El Hasnaoui A, Chicoye A, et al. Place de la prévention dans la politique de santé. Montrouge : John Libbey Eurotext, 2007.

22. Jonas H. Philosophical essays: from ancient creed to technological man. Prentice-Hall (NJ) : Englewood Cliffs, 1974.

23. Andorno R. The right not to know: an autonomy based approach. J Med Ethics $2004 ; 30: 435-9$.

24. Vassoler F.M., White SL, Schmidt HD. Epigenetic inheritance of a cocaineresistance phenotype. Nat Neurosci $2013 ; 16: 42-7$.

25. Bale TL, Baram TZ, Brown AS. Early life programming and neurodevelopmental disorders. Biol Psychiatry 2010 ; 68 : 314-9.

26. Posner RA. The right of privacy. Georgia Law Rev 1978 ; $393: 411-4$.

27. Heckman JJ. The developmental origins of health. Health $\varepsilon$ con $2012 ; 21$ : 24-9.

28. 0 'Campo P. Are we producing the right kind of actionable evidence for the social determinants of health? J Urban Health 2012 ; 89 : 881-93.

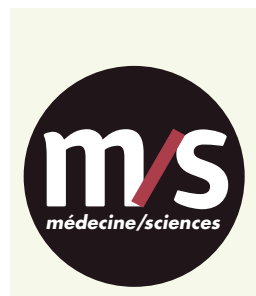

Tarifs d'abonnement $m / s-2016$

Abonnez-vous

à médecine/sciences
$>$ Grâce à $\mathrm{m} / \mathrm{s}$, vivez en direct les progrès des sciences biologiques et médicales

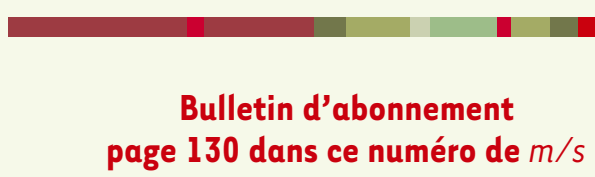

TIRÉS À PART

દ. Rial-Sebbag 